\title{
Numérique et médecine de la douleur
}

\author{
R. Dallel \\ (C) Lavoisier SAS 2021
}

L'intelligence artificielle et le numérique sont entrés dans le domaine de la santé et y joueront un rôle croissant. Nous devons redoubler d'efforts pour faire que la société profite au mieux de ces avancées technologiques. L'innovation numérique et l'intelligence artificielle peuvent ainsi contribuer à améliorer les soins, la formation, l'information et la recherche dans le domaine de la douleur.

Dans le domaine du soin, les soignants utilisent de plus en plus les nouvelles technologies pour s'informer et aider aux choix diagnostiques et thérapeutiques. Les outils numériques paraissent aussi capables de fluidifier le " parcours patient », de le rendre plus attractif et personnalisé. C'est également une solution pour améliorer l'organisation des soins. Par exemple, télémédecine et télédiagnostic peuvent accroître l'efficacité des consultations médicales et offrir une solution au problème des déserts médicaux. Ainsi, le renseignement en ligne par le patient de ses ressentis, la communication directe de ses données cliniques à l'aide d'objets connectés aideront le médecin à mieux évaluer l'état de son patient, d'autant que ce dernier est en situation réelle, et non pas en consultation avec ses échanges verbaux informels et le renseignement fastidieux de questionnaires. Inversement, des outils numériques d'éducation à la santé, à la douleur en général seront des supports importants du soin, en améliorant les compétences du patient à assumer sa douleur.

Dans le domaine de la recherche sur la douleur, les technologies numériques permettront des progrès importants en croisant de grandes quantités de données cliniques, biologiques, voire génétiques, afin, par exemple, d'identifier les patients à risque de développer des douleurs chroniques ou les meilleurs candidats à un traitement antalgique. Des modèles mathématiques doivent être développés pour se substituer progressivement aux critères approximatifs actuellement utilisés pour évaluer l'efficacité des traitements. Par ailleurs, la mise en évidence fortuite de corrélations par l'analyse au hasard et le traitement par l'intelli-

R. Dallel $(\bowtie)$

Faculté de Chirurgie Dentaire,

2 rue de Braga, 63100 Clermont-Ferrand, France

Université Clermont Auvergne, CHU Clermont-Ferrand, Inserm,

Neuro-Dol, F-63000 Clermont-Ferrand, France

e-mail : radhouane.dallel@uca.fr gence artificielle de données de santé généreront des hypothèses originales, les niveaux de preuves étant ensuite validés par des études dédiées.

Dans le domaine de la formation, les établissements d'enseignement mettent en place des cursus spécifiques pour former les futurs soignants à l'usage de ces nouvelles technologies, devenues indispensables à l'exercice de leur métier. En effet, de nombreux métiers de la santé seront impactés par ces nouvelles technologies : de nouveaux emplois seront créés, d'autres disparaîtront, beaucoup devront s'adapter.

Ce numéro spécial rassemble des experts de premier plan en santé numérique dans le domaine de la douleur. À travers quatre articles originaux, les auteurs font ici un tour d'horizon complet sur des sujets cruciaux dans le domaine de la douleur, la place de la santé numérique dans le diagnostic et le traitement de la douleur chronique, y compris chez le patient dyscommunicant.

Duvaux et al. [1] réalisent une synthèse des travaux sur la place de la télémédecine (téléexpertise, téléconsultation, télésoin, télésurveillance) dans la prise en charge de la douleur chronique. Ils soulignent que la technologie, avec ses limites, ne doit pas être une finalité, mais un outil au service d'une organisation plus fluide et mieux partagée du parcours des patients : elle doit ainsi permettre d'expérimenter de nouveaux usages dans une coopération ville-hôpital repensée. Les auteurs proposent aussi des pistes concrètes susceptibles de faciliter l'adoption d'une « doctrine d'usage » de la télémédecine dans le champ de la douleur chronique et, ainsi, d'en accélérer l'usage.

Les technologies numériques semblent être une solution pour mieux caractériser, surveiller et gérer la douleur et ses comorbidités. Elles facilitent les interventions à distance, palliant ainsi les difficultés inhérentes aux déplacements sur de longues distances ou aux visites régulières chez le médecin, généraliste ou spécialiste. Kerckhove et al. [2] dressent un état des lieux des travaux évaluant l'utilisation et l'impact de la santé numérique sur des patients souffrant de douleurs chroniques. Ils évaluent les text-messages, les carnets électroniques et les applications mobiles. Enfin, les auteurs discutent les limites et défis futurs de l'intégration de l'e-santé dans la prise en charge des douleurs chroniques. Ils suggèrent que l'intégration de l'e-santé est prometteuse pour 
la prise en charge de ces patients, mais soulignent la nécessité d'effectuer des études rigoureuses pour en évaluer le bénéfice réel.

Il n'existe pas aujourd'hui d'examen paraclinique ou de méthode fiable capable d'objectiver la présence ou l'absence d'une douleur et encore moins son intensité. Parce que la douleur est une expérience personnelle, subjective, c'est l'autoévaluation par le patient douloureux lui-même qui apporte la mesure la plus fiable de cette douleur, le plus souvent en utilisant une échelle verbale, visuelle analogique ou numérique. Cependant, cette autoévaluation est difficile, voire impossible, chez des patients non communicants : principalement les enfants en dessous de six ans, les adultes avec des difficultés de langage, une confusion mentale ou des troubles de la conscience et les personnes âgées présentant une altération des fonctions cognitives. L'expression du visage représente un mode majeur de communication non verbale des émotions. C'est d'ailleurs le paramètre le plus utilisé dans les échelles d'hétéroévaluation de la douleur. Les progrès des technologies numériques de reconnaissance faciale, capables de reconnaître automatiquement et de mesurer les mouvements faciaux, offrent l'opportunité d'analyser l'expression faciale en s'affranchissant des biais d'interprétation liés au facteur humain. Fontaine et Santucci-Sivolotto [3] analysent le potentiel de la reconnaissance automatisée de l'expression faciale en pratique clinique pour estimer, de manière relativement objective, l'intensité de la douleur ressentie par les patients dans diverses situations cliniques.

Le numérique ouvre également de nouvelles perspectives dans le traitement de la douleur. Les thérapies numériques proposent des interventions thérapeutiques pilotées par des logiciels de grande qualité pour prévenir, gérer ou traiter un trouble médical ou une maladie. Ces solutions numériques peuvent être utilisées seules ou combinées à un médicament, un dispositif médical ou une thérapie afin d'optimiser les effets du traitement. De toutes les approches numériques pour le traitement de la douleur, la réalité virtuelle fait l'objet depuis plusieurs années d'études et de tests sur différents types de douleurs, dont les résultats semblent prometteurs. Dans leur article, Villemin et al. [4] proposent une revue de l'utilisation de la réalité virtuelle, de son origine et de son fonctionnement.

En conclusion, nous remercions tous les auteurs qui ont accepté de partager leurs connaissances sur l'e-santé en médecine de la douleur dans le cadre de ce numéro spécial. Le numéro rassemble un ensemble original d'articles d'opinions et de critiques perspicaces qui tentent d'aborder plusieurs dimensions de la douleur chronique. Nous espérons que ce numéro permettra non seulement d'améliorer notre compréhension de la douleur et de sa gestion, mais aussi de stimuler les innovations du numérique en santé liée à la douleur.

\section{Références}

1. Duvaux C, Gobin M, Nizard J (2021) Télémédecine et prise en charge de la douleur chronique en 2021 - mise en perspective. Douleur Analg 34(3)

2. Kerckhove N, Corteval A, Eschalier A (2021) Apport du numérique dans la prise en charge médicale des patients souffrant de douleurs chroniques. Douleur Analg 34(3)

3. Fontaine D, Santucci-Sivolotto S (2021) Évaluer la douleur par reconnaissance automatique de l'expression faciale : un espoir illusoire ou la réalité pour demain ? Douleur Analg 34(3)

4. Villemin C, Abel F, Dispersyn G, et al (2021) La réalité virtuelle comme antidouleur : une revue systématique de la littérature. Douleur Analg 34(3) 